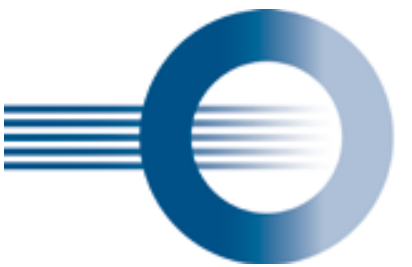

\section{AG Ultraschall}

in der Deutschen Röntgengesellschaft

\title{
Kontrastmittelsonografie (CEUS) in der Radiologie: Eine erste Analyse und kritische Betrachtung der AGUS
}

\section{Zusammenfassung:}

$\nabla$

Moderne Ultraschallverfahren bieten Möglichkeiten zur dynamischen Erfassung der Mikrovaskularisation mit der Kontrastmittelsonografie (CEUS), eine Erfassung der Gewebemorphologie mit der Elastografie, 3D- und 4D-Darstellungen und Fusionen mit der Computertomografie (CT) und Magnetresonanztomografie (MRT). Die noch junge Arbeitsgemeinschaft für Ultraschall in der Radiologie (AGUS) hat sich zum Ziel gesetzt, diese modernen Ultraschallverfahren als Bestandteil der radiologischen Bildgebung und Intervention zu stärken.

\section{Abstract: \\ $\nabla$}

Modern diagnostic ultrasound enables dynamic evaluation of microvascularization using contrast enhanced ultrasound (CEUS), characterization of the tissue morphology using elastografy, 3D- and $4 \mathrm{D}$-imaging, and image fusion with cross sectional imaging techniques such as computed tomografy (CT) and magnetic resonance imaging (MRI), respectively. The recently founded working group on radiological ultrasound (AGUS) has the intention to integrate these modern ultrasound-techniques into both diagnostic and interventional procedures.

\section{Einleitung: \\ $\nabla$}

Die Hochleistungsultraschalldiagnostik ermöglicht uns eine immer detailgenauere Betrachtung mit der Real-time Betrachtung von dynamischen Prozessen. Mit der Kontrastmittelsonografie (CEUS) eröffnen sich durch die digitale Erfassung von mik- rovaskulären Prozessen neue Aspekte einer Beurteilung der Mikrozirkulation bis zu den Kapillaren [1-5]. Dies wird schon jetzt genutzt, um insbesondere Lebertumore mit wesentlich höherer Genauigkeit zu detektieren und zu charakterisieren, als dies mit der B-Bild-Sonografie oder Duplexverfahren möglich ist. Es ergeben sich neue Möglichkeiten einer Interventionsplanung, für ein periinterventionelles Monitoring und eine postinterventionelle Kontrolle [6-8]. Fast kein Bereich der vaskulären und Tumor-Diagnostik steht bezüglich der diagnostischen Möglichkeiten von CEUS derzeit nicht im Blickpunkt des wissenschaftlichen

Interesses der Ultraschall-durchführenden Ärzte. Es stellt sich aber die kritische Frage, inwieweit das Potenzial des CEUS Eingang in die radiologische Bildgebung gefunden hat und wie die Durchdringung innerhalb der Radiologie auf absehbare Zeit erfolgen wird.

Zu Beginn einer kritischen Betrachtung in der radiologischen Bildgebung scheinen die folgenden Punkte entscheidend für die weitere Analyse:

- Formulieren einer Vision: Wie soll der Stellenwert von CEUS in der Radiologie sein?

- Wo bestehen derzeit Engpässe?

- Wie lässt sich CEUS in den Radiologischen Instituten etablieren?

Auf die Frage, inwieweit die Radiologie in Deutschland bezüglich CEUS Anschluss an die Entwicklung der die Guidelines bestimmenden Fachdisziplinen hält, fällt die Bilanz eher kritisch aus. So kann der Eindruck entstehen, dass bezüglich CEUS der Anschluss verpasst wurde. Legt man alleine die aufgrund aktueller Verkaufszahlen erhobenen möglichen Untersuchungs- zahlen zugrunde, so werden bis zu 95\% der CEUS-Untersuchungen nicht von Radiologen durchgeführt, und wenn CEUS in der Radiologie durchgeführt wird, dann meist nur an speziellen Zentren und von ausgewiesenen Spezialisten.

So findet sich bei einem 1. Expertenmeeting eine breite Zustimmung zur These: Die meisten Radiologen sind dabei, den Anschluss an die Ultraschall/ CEUS-Entwicklung als ergänzende Verfahren zu verlieren oder haben ihn schon verloren.

Dennoch finden sich Ansatzpunkte, die dieser These widersprechen und die Chancen für eine differenzierte Betrachtung im Detail eröffnen.

- Die wissenschaftliche Aktivität auf dem Gebiet der CEUS in der Radiologie ist fester Bestandteil zur Etablierung von CEUS als dynamische Bildgebung der Mikrovaskularisation.

- Spezielle technische Entwicklungen wie Fusionsbildgebungen mit der CT und MRT sind geradezu prädisponiert, die radiologische Dokumentation zu erweitern.

- Wenn auch von ausgewiesenen Experten, so kann doch in Spezialanwendungen innerhalb der Radiologie derzeit schon das Spektrum insbesondere der vaskulären Bildgebung entscheidend erweitert und entsprechend integriert werden.

- Gerade in interdisziplinären Ultraschallzentren wird auch für CEUS die Expertise von Radiologen geschätzt.

Ein klarer Appell muss sein, die Zukunft von Ultraschall/CEUS selber und aktiv zu gestalten und die Radiologie bzw. die Radiologen nicht nur einzubinden, sondern einer aktiven Rolle zuzuführen. Denn die schlechteste aller Möglichkeiten wäre, die großen diagnostischen Möglichkeiten von CEUS nicht auch als komplementäre Bildgebung in der Radiologie zu nutzen. Gerade der Aspekt, dass CEUS bei Verwendung von sulphur hexafluoride microbubbles (SonoVue®/BRACCO) eine Kontrastmittelbildgebung ohne Gefährdung der Nierenfunktion ermöglicht, kann ganz entscheidende diagnostische Vorteile bieten [9].

Um die Bedeutung von CEUS in der Radiologie zu stärken, ist eine Strategie erforderlich, die sich an den (Management)Zielen der Radiologen im Umgang mit Ultraschall/CEUS orientiert. Basis bilden die 
Vorteile/Nutzen der Ultraschall/CEUSTechnologie in der Radiologie. Alle Strategien dürfen nicht isoliert gesehen werden - sie orientieren sich an ihrem Beitrag zur Diagnosefindung.

Das Ziel für den Patienten einen bestmöglichen Nutzen zu erreichen, ist nur dann gegeben, wenn es gelingt, auch unter Berücksichtigung ökonomischer Aspekte eine Bildgebung mit CEUS mit hohem diagnostischem Potenzial und hoher Qualität innerhalb der Radiologie zu positionieren. Dies beinhaltet auch die Akzeptanz der möglichen Überweiser.

Der Nutzen einer qualitativ hochwertigen Diagnostik mit der Sonografie kann für die Radiologie in den Aspekten Detailgenauigkeit, hoher Bildqualität, einem breitem Einsatzspektrum, optimaler Patientenversorgung mit im günstigsten Fall verkürzten Liegezeiten, Diagnosesicherheit und Ökonomisierung liegen. Damit ist auch eine NICE-Empfehlung (National Institute for Health and Care Excellence) zu beachten.

Um die Erwartungen erfüllen zu können, eine Diagnostik mit der hochauflösenden Technik der Kontrastmittelsonografie (CEUS) in der Radiologie zu etablieren, gilt es, Ziele zu definieren, wie dies zu erreichen ist. Dazu gehört, insgesamt die Position der Ultrasschalldiagnostik zu stärken. CEUS erreicht dann eine wichtige Stellung, wenn es gelingt, mit hohem Qualitätsanspruch auch schwierige diagnostische Fragestellungen beantworten $\mathrm{zu}$ können, was zur Bezeichnung einer Premium-Schalluntersuchung führen kann. Über qualifizierte Ausbilder ist dann längerfristig anzustreben, CEUS als RoutineUntersuchung verfügbar zu machen.

Es ist eine große Herausforderung, die hohen diagnostischen Möglichkeiten, die CEUS bietet, auch in der Radiologie zu etablieren. Widerstände begründen sich zunächst einmal in der derzeit noch schlechten Verfügbarkeit und der geringen Vergütung einer technisch und personell aufwendigen Untersuchungsmodalität bzw. auch Dienstleistung. Kritisch wird die Bilddokumentation betrachtet, die einen hohen Datenaufwand bei Cine-Sequenzen erforderlich macht. Ebenso kritisch wird die Untersucherabhängigkeit und Reproduzierbarkeit betrachtet. Da der Stellenwert von CEUS zunimmt, besteht auch der Aspekt der Verdrängung anderer bildge- bender Verfahren wie CT/MRT. Derzeit bietet CEUS innerhalb der radiologischen Weiterbildung und Fortbildung kaum Möglichkeiten, sich einen Karierrevorteil zu verschaffen, da die Bildgebung insgesamt noch wenig postiv innerhalb der Radiologie beurteilt wird. Dies liegt auch daran, dass eine spezielle Technik erforderlich ist, die in den meisten derzeit noch routinemässig verfügbaren Ultraschallgeräten nicht verfügbar ist.

Die entscheidenden Widerstände gegenüber CEUS bestehen derzeit in:

- einer fehlenden leistungsbezogenen Vergütung,

- eine ambivalente Einstellung zu CEUS innerhalb der Radiolgie,

- ungeklärten berufspolitischen Fragen,

- einem hohen Zeit- und Arbeitsaufwand,

- einem derzeit noch schlechten Image.

Die nächsten Schritte, die dazu beitragen können, die Bedeutung von CEUS in der Radiologie zu stärken, können in einer Imageaufbesserung bestehen, verbunden mit einer optimierten, möglichst standardisierten Dokumentation, dem Aufbau von interdisziplinären Kooperationen, so in möglichst zentralen Ultraschallzentren, aber auch mithilfe von Netzwerken. Die Kostenfrage lässt sich nur mithilfe einer medizinisch-ökonomischen Analyse klären, wobei erste Ergebnisse auch interdisziplinärer Ultraschallzentren darauf hindeuten, dass CEUS zur effektiven Patientenversorgung beitragen kann.

Entscheidend für einen Imagezugewinn von CEUS innerhalb der Radiologie ist eine qualitativ hochwertige Ausbildung. Damit kommt entsprechenden Ausbildungsmodulen eine entscheidende Bedeutung zu, die es dann ermöglichen, diese Bildgebung mit hoher diagnostischer Aussagekraft umfassend anzubieten und entsprechend attraktiv für Zuweiser und Mitarbeiter zu gestalten.

Innerhalb der Deutschen Röntgengesellschaft (DRG) stellt die Etablierung einer Arbeitsgemeinschaft für Ultraschall in der Radiologie (AGUS) einen Schritt dar, die Bedeutung von Ultraschall und CEUS als bildgebende Modalität zu unterstreichen. Dabei wird die Bedeutung von Ultraschall auch durch die aktuellen technischen Entwicklungen innerhalb der radiologischen Bildgebung entscheidend gestärkt, insbesondere auch im Hinblick auf die digitale Datenverarbeitung. Bereits angedachte
Ausbildungsmodule sollen bei zukünftigen Kongressen berücksichtigt werden. Durch zusätzliche Spezialkurse soll insbesondere CEUS zunehmend intensiv geschult werden und damit weitere Verbreitung finden.

Die Möglichkeiten, die CEUS bietet, sind umfassend und werden in aktuellen Guidelines bezüglich der Leberdiagnostik und bei extrahepatischen Anwendungen bereits in der Breite angedacht [1,2]. Die Ergebnisse von Multicenterstudien deuten eine hohe diagnostische Sicherheit von CEUS bezüglich der Detektion und Charakterisierung von Lebertumoren an. Auch scheinen die diagnostischen Möglichkeiten von CEUS bezüglich der Detektion und Charakterisierung von Endoleaks unbestritten.

Dennoch bleibt kritisch zu beachten, dass zum jetzigen Zeitpunkt bezüglich einer Vielzahl von Anwendungen von CEUS das diagnostische Potenzial auf wenige erfahrene Untersucher beschränkt bleibt, die Anwendungen meist nur umfassend in wenigen Ultraschallzentren verfügbar sind und außer bezüglich der Lebertumordiagnostik noch Multicenterstudien fehlen, die den diagnostischen Stellenwert der Methodik definieren. Dies sind breite Aufgabenfelder für die Zukunft.

\section{Literatur:}

1 Claudon M, Dietrich CF, Choi BI et al. Guidelines and good clinical practice recommendations for Contrast Enhanced Ultrasound (CEUS) in the liver - update 2012: A WFUMBEFSUMB initiative in cooperation with representatives of AFSUMB, AIUM, ASUM, FLAUS and ICUS. Ultrasound in medicine $\&$ biology 2013; 39: 187-210

2 Claudon M, Dietrich CF, Choi BI et al. Guidelines and good clinical practice recommendations for contrast enhanced ultrasound (CEUS) in the liver--update 2012: a WFUMBEFSUMB initiative in cooperation with representatives of AFSUMB, AIUM, ASUM, FLAUS and ICUS. Ultraschall in der Medizin 2013; 34: 11-29

3 Clevert DA, D‘Anastasi M, Jung EM. Contrastenhanced ultrasound and microcirculation: efficiency through dynamics--current developments. Clinical hemorheology and microcirculation 2013; 53: 171-186

4 Fischer T, Sack I, Thomas A. Characterization of focal breast lesions by means of elastografy. RoFo : Fortschritte auf dem Gebiete der Rontgenstrahlen und der Nuklearmedizin 2013; 185: 816-823

5 Jung EM, Wiggermann P, Stroszczynski C et al. [Ultrasound diagnostics of diffuse liver diseases]. Der Radiologe 2012; 52: 706-716 
6 Clevert DA, Jung EM. [Interventional sonografy of the liver and kidneys]. Der Radiologe 2013; 53: 962-973

7 Gurtler VM, Sommer WH, Meimarakis G et al. A comparison between contrast-enhanced ultrasound imaging and multislice computed tomografy in detecting and classifying endoleaks in the follow-up after endovascular aneurysm repair. Journal of vascular surgery 2013; 58: 340-345

8 Strobel D, Bernatik T, Blank $W$ et al. Diagnostic accuracy of CEUS in the differential diagnosis of small $(</=20 \mathrm{~mm})$ and subcentimetric $(<1=10 \mathrm{~mm})$ focal liver lesions in comparison with histology. Results of the DEGUM multicenter trial. Ultraschall in der Medizin 2011; 32: 593-597
9 Greis C. [Summary of technical principles of contrast sonografy and future perspectives]. Der Radiologe 2011; 51: 456-461

Jung EM, Stroszczynski C, Institut für Röntgendiagnostik Universitätsklinikum Regensburg Franz-Josef-Strauß-Allee 11

93053 Regensburg

christian.stros@ukr.de

$\mathrm{Tel}+499419447400 / 7401$

Fax +499419447402 